\title{
Persistence of Clostridium botulinum Neurotoxin Type E in Tissues from Selected Freshwater Fish Species: Implications to Public Health
}

\author{
ADAM M. YULE, ${ }^{1}$ JOHN W. AUSTIN, ${ }^{2}$ IAN K. BARKER,${ }^{3}$ BRIGITTE CADIEUX,${ }^{2}$ AND RICHARD D. MOCCIA ${ }^{1 *}$ \\ ${ }^{1}$ Aquaculture Centre, Department of Animal and Poultry Science, and ${ }^{3}$ Canadian Cooperative Wildlife Health Centre, Department of Pathobiology, \\ Ontario Veterinary College, University of Guelph, Guelph, Ontario, Canada N1G 2W1; and ${ }^{2}$ Microbial Research Division, Bureau of Microbial \\ Hazards, Food Directorate, Health Products and Food Branch, Health Canada, Tunney's Pasture, Ottawa, Ontario, Canada K1A OL2
}

MS 05-208: Received 30 April 2005/Accepted 13 November 2005

\begin{abstract}
Rainbow trout (Oncorhynchus mykiss), round gobies (Neogobius melanostomas), yellow walleye (Stizostedion vitreum), and yellow perch (Perca flavescens) were given Clostridium botulinum neurotoxin type E (BoNT/E) at four doses (0, 800, 1,500 , and 4,000 mouse lethal doses). BoNT/E was sought in the fish tissues at death or at the conclusion of the experiment (10 days after treatment). Fish were divided into a "fillet" (axial musculature) and a "nonfillet" sample before testing for BoNT/E toxicity with a mouse bioassay. BoNT/E was detected in all species. The percentage of positive BoNT samples ranged across the species and doses from 0 (trout, perch, and walleye) to $17 \%$ (round goby) in fillet tissues and from 0 (perch) to $92 \%$ (round goby) in nonfillet tissues. The lack of positive fillet samples in three key commercial fish species suggests that the public health implications of eating these fish are minimal. However, the presence of toxin in the nonfillet compartment of a high proportion of fish supports the hypothesis that live intoxicated fish are a vehicle for the transfer of BoNT/E to fisheating birds, which are then in turn, intoxicated.
\end{abstract}

Foodborne botulism has been recognized for more than 1,000 years; however, according to Dolman (3), Van Ermengen published the first true clinical description of the disease in 1897. Foods that have been historically associated with foodborne botulism are low-acid canned foods; meats and vegetables; smoked, salted, or cured meats; and fish (8). The close association between fish products and type $\mathrm{E}$ botulism has long been recognized. The first known case of type $\mathrm{E}$ botulism in humans resulting from the consumption of fish derived from the Great Lakes was reported in 1960 (11). The outbreak was traced back to vacuumpacked smoked ciscoes (Coregonus artedi). Testing isolated type E Clostridium botulinum toxin from samples of the fish heads, fins, and bones (5). In this case, like in the majority of foodborne botulism cases, there was postmortem toxin production prior to the fish being consumed.

C. botulinum persists in the form of dormant spores when environmental conditions are adverse. These spores are found in freshwater, marine, and terrestrial environments throughout the world and can remain viable for years (9). Under appropriate conditions, the spores germinate, the bacterium proliferates, and toxin can be produced. Since the first report of the disease, the food industry has become aware of foodborne botulism risks and has taken appropriate steps to ensure their control. However, occasional out-

* Author for correspondence. Tel: 519-824-4120, Ext 56216; Fax: 519767-0573; E-mail: rmoccia@uoguelph.ca. breaks still occur. Recently, there has been concern that live fish in the Great Lakes may have $C$. botulinum neurotoxin type $\mathrm{E}(\mathrm{BoNT} / \mathrm{E})$ present in their tissues, thereby posing a public health hazard (1).

Since 1999, large-scale type E botulism epizootics have occurred in fish-eating birds on the Great Lakes, primarily Lake Erie. What is unique about these outbreaks is that the avian species mainly involved are known to consume only live fish, and it is hypothesized that the live fish are vectoring the toxin from its point of origin (most likely present as preformed free toxin in the food items eaten by these fish, such as in other smaller fish, invertebrates, sediment, or decaying organic material) to the birds $(14,15)$. To fully understand the potential risk of consuming fish caught during type E botulism epizootics, and if the fish are vectoring toxin to the birds, it is necessary to determine if free $\mathrm{BoNT} / \mathrm{E}$ is present in fish that have been exposed to BoNT/E. For the purposes of this article, free BoNT/E is defined as BoNT/E present in the fish that is not bound to a receptor and is therefore detectable by mouse bioassay. Toxin that is not free and is not detectable does not pose a human health risk. The presence of free BoNT/E in fish could pose a public health concern and would support the hypothesis that live fish are transferring toxin to fish-eating birds. In 1995 alone, an estimated 96,000 anglers fished on Lake Erie, catching over three million fish (7). Of those three million fish, roughly 306,000 walleye, 556,000 perch, and 41,000 rainbow trout were kept for consumption (7). 
There is also a very large commercial fishery on Lake Erie. In 2002, in U.S. waters alone, over 1.4 million pounds of yellow perch were caught and processed (10).

The intent of this study was to determine if BoNT/E was present postmortem in four common Lake Erie fish species following acute exposure to BoNT/E at four toxin doses. This work provided further insight into the possible public health risks associated with the consumption of fish caught during botulism epizootics and further assessed the role of live fish in vectoring BoNT/E to fish-eating birds.

\section{MATERIALS AND METHODS}

Fish acquisition and holding. This research project design followed the University of Guelph Animal Care Committee, Animal Use Protocol \#02R161. The four species used were juvenile domesticated rainbow trout $(214.5 \pm 3.4 \mathrm{~g}$ standard error [SE] $)$ acquired from the Alma Aquaculture Research Station (Alma, Ontario, Canada), round gobies (Neogobius melanostomas) (75.7 \pm 2.3 g) caught in Lake Erie by the Ontario Ministry of Natural Resources trawler (Erie Explorer, Port Dover, Ontario, Canada), pond-raised walleye (Stizostedion vitreum) $(61.2 \pm 3.0 \mathrm{~g})$ acquired from Leonard Walleye Culture (Kingston, Ontario, Canada), and pond-raised yellow perch (Perca flavescens) (88.0 \pm 3.5 g) from Cooper Bay Fisheries (Selkirk, Ontario, Canada).

Each species was held in individual 350 -liter, $0.35-\mathrm{m}^{3}$ tanks (1.1 by 1.1 by $0.29 \mathrm{~m}$ ) supplied with continuously flowing freshwater with excellent water chemistry characteristics. Flow rates were maintained at 30 liter $\mathrm{min}^{-1}$ to ensure stable water quality. A photoperiod of $12 \mathrm{~h}$ dark: $12 \mathrm{~h}$ light was provided with an automated, shielded fluorescent lighting system. Prior to experimentation, fish were fed a size six-pt regular sinking pellet feed (Martin Mills Inc., Elmira, Ontario, Canada). All fish were quarantined in the laboratory for a minimum of 2 months prior to experimentation.

BoNT/E production. C. botulinum type E (strain Russ) was grown for 4 days in special peptone, peptone, glucose, and yeast extract $(5 \%$ special peptone, Oxoid, Basingstoke, Hampshire, England) in an anaerobic chamber (Coy Laboratory Products Inc., Grass Lake, Mich.) at $30^{\circ} \mathrm{C}$. After 4 days, the whole culture was centrifuged (RC-5 superspeed refrigerated centrifuge, Sorvall, Wilmington, Del.) at $20,000 \times g$ for $30 \mathrm{~min}$ at $4^{\circ} \mathrm{C}$, and the supernatant was harvested. Samples were trypsinized $(0.14 \%$ trypsin, wt $/ \mathrm{vol}$ ) at $37^{\circ} \mathrm{C}$ for $1 \mathrm{~h}$ (trypsin 250 , Becton Dickinson, Sparks, Md.) (2). The culture supernatant was then twofold serially diluted, and each dilution was tested for toxicity by mouse bioassay $(2$ mice $\times 5$ dilutions $=10$ mice total). The highest dilution that is lethally toxic in mice is equal to one mouse lethal dose (MLD) per $0.5 \mathrm{ml}^{-1}$ (because $0.5 \mathrm{ml}$ was injected into the mouse). MLD rather than 50\% MLD was used to express toxin concentration because toxicity was based on death being observed in both inoculated mice and not in just one of the two. The reciprocal of the dilution factor was corrected to $1 \mathrm{ml}$ and was used to describe the concentration of toxin present in the original supernatant.

For the purposes of dosing, the volume of supernatant that fit conveniently into a no. 5 gelatin capsule (Gaines Nutrition, Santee, Calif.) was $100 \mu$ l. Thus, because the MLD per milliliter of the original supernatant was 400 MLD, it could then be concentrated $2 \times$ to achieve 800 MLD per $100 \mu 1,3.75 \times$ to achieve 1,500 MLD per $100 \mu \mathrm{l}$, and $10 \times$ to achieve 4,000 MLD per 100 $\mu 1$, using $0.45-\mu \mathrm{m}$ centrifugal filters (Whatman, Clifton, N.J.) with a molecular weight cutoff of 30 . For the purposes of the negative control (0 MLD) capsules, supernatant was treated with specific $C$. botulinum type E monovalent antiserum (Aventis, Toronto, Ontario, Canada) to neutralize toxin activity.

No. 5 gelatin capsules were $75 \%$ filled with gelatinized corn starch (National 1215, Brampton, Ontario, Canada), and $100 \mu \mathrm{l}$ of the trypsinized supernatant was added. Capsules were immediately frozen in a conventional freezer and maintained at a temperature of $-20^{\circ} \mathrm{C}$ until the time of gastric intubation.

Experimental design. The fish were held individually at $19.3^{\circ} \mathrm{C} \pm 0.1^{\circ} \mathrm{C}$ in twelve 90 -liter aerated aquaria. Fish were fasted for 6 days prior to experimentation to empty their digestive systems and allowed to acclimate in the experimental aquaria for a final 3 days of fasting. Experimental fish were also not fed for the duration of the experiment.

Fish were individually placed into a $0.42 \mathrm{~g} \mathrm{liter}^{-1}$ tricaine methanesulfate (MS-222) anesthetic bath. Once the fish had reached stage four anesthesia (13), they were removed. A capsule was placed into the distal end of a 6-mm-diameter rigid tygon tube. The tube was slowly passed through the oesophageal sphincter and into the stomach of the fish. Once the end of the tube was inside the stomach, a tube of smaller diameter was advanced down the bore of the outer tube, forcing the capsule into the stomach of the fish. Then, fish were immediately returned to the experimental tanks for recovery.

Sample handling. The majority of fish expressed clinical signs that included equilibrium loss, pigmentation change, respiratory distress, and erratic swimming. A description of the onset of clinical signs and the number of fish expressing clinical signs is outlined in Yule et al. (14). After death (as a result of BoNT/E intoxication, or, in the case of fish surviving to 10 days, by an overdose of tricaine methanesulfate MS-222), experimental fish were immediately double bagged and frozen at $-20^{\circ} \mathrm{C}$ in a conventional freezer. Fish were stored in a conventional freezer at $-20^{\circ} \mathrm{C}$ until testing ( 1 to 3 weeks later).

Preparation of samples. Prior to testing, fish were allowed to thaw entirely by being held at $4^{\circ} \mathrm{C}$ overnight. The fish were then incised longitudinally on the ventral abdominal wall to facilitate the removal of the entire visceral mass, including kidneys. The heads of the fish were then cut off at the posterior margin of the gills, and the tail was cut off just anterior to the caudal peduncle. The remainder of the fish was rinsed with tap water and stored at $4^{\circ} \mathrm{C}$ until it was processed the next day. This portion of the fish was considered the fillet sample, and it consisted largely of flank musculature. The head and tail, combined with the viscera, was considered the nonfillet sample. The nonfillet sample was then placed in a plastic bag and stored at $4^{\circ} \mathrm{C}$ until processed (within 1 week). The fillet fractions were weighed (Mettler PJ3000, Mettler Toledo, Mississauga, Ontario, Canada), and an equal weight of $0.2 \%$ gelatin in a sodium phosphate buffer, $\mathrm{pH}$ 6.2, was added. The fillets were then blended (Osterizer blender, Sunbeam, Boca Raton, Fla.) at high speed for $30 \mathrm{~s}$ and centrifuged at $15,000 \times g$ for $20 \mathrm{~min}$ at $4^{\circ} \mathrm{C}$. The same procedure was conducted with the nonfillet samples. The supernatants were filter sterilized $(0.45 \mu \mathrm{m})$ and stored at $4^{\circ} \mathrm{C}$ until tested for toxicity the same day.

Toxin confirmation and mouse bioassay. Supernatants were tested for the presence of BoNT/E as described by Austin and Blanchfield (2). Briefly, each extract $(1.5 \mathrm{ml})$ was trypsinized for $1 \mathrm{~h}$ at $37^{\circ} \mathrm{C}$, after which $0.55 \mathrm{ml}$ was injected intraperitoneally into each of two 20-g mice (Charles River Canada, Quebec, Canada) per sample. Mice were observed for signs of botulism for up to $72 \mathrm{~h}$. Samples found to be toxic by mouse bioassay were sub- 
TABLE 1. Percentage of BoNT/E-positive fillet and nonfillet samples from rainbow trout, round goby, walleye, and yellow perch following experimental exposure to 800, 1,500, and 4,000 MLD of BoNT/E at $19.3^{\circ} \mathrm{C}$

$\%$ positive (no./total no.)

\begin{tabular}{|c|c|c|c|c|c|}
\hline Sample & MLD & Rainbow trout & Round goby & Walleye & Yellow perch \\
\hline \multirow[t]{2}{*}{ Fillet } & 800 & $0(0 / 12)$ & $0(0 / 12)$ & $0(0 / 12)$ & $0(0 / 12)$ \\
\hline & 1,500 & $0(0 / 12)$ & $0(0 / 12)$ & $0(0 / 12)$ & $0(0 / 12)$ \\
\hline \multirow[t]{2}{*}{ Nonfillet } & 800 & $8(1 / 12)$ & $92(11 / 12)$ & $42(5 / 12)$ & $0(0 / 12)$ \\
\hline & 1,500 & $17(2 / 12)$ & $75(9 / 12)$ & $67(8 / 12)$ & $17(2 / 12)$ \\
\hline
\end{tabular}

sequently neutralized with specific $C$. botulinum type E monovalent antiserum, and mouse inoculation was repeated as above. Failure to observe signs consistent with botulism in these mice confirmed that signs in the originally injected mice were due to BoNT/E.

The majority of samples found to be toxic by mouse bioassay were confirmed as BoNT/E by the neutralization test. To reduce the number of mice used, not all toxic samples underwent conformational neutralization tests. Since all toxic samples (28 of 28) that were subsequently neutralized and retested in mice were confirmed as BoNT/E, toxic samples that were not confirmed by neutralization were assumed to be BoNT/E.

\section{RESULTS AND DISCUSSION}

Nonfillet samples. Nonfillet samples containing free BoNT/E were detected in all species, varying from $0 \%$ in the 800 MLD yellow perch to $92 \%$ in the 800 MLD round gobies (Table 1). Although a high proportion of the nonfillet samples were positive, this portion of the fish is not normally consumed by people and therefore is regarded to be of minimal public health risk.

Fillet samples. Free toxin in the fillet (axial muscle) fraction was used to assess human health risks because it is the most commonly consumed part of the fish. Overall, in this study, a very small proportion of fillet samples tested contained detectable BoNT/E (0 of 36 rainbow trout, 2 of 36 round gobies, 0 of 36 of walleye, 0 of 36 yellow perch) (Table 1). Both positive goby samples were from fish administered the highest dose of toxin (4,000 MLD).

Public health implications. The high proportion of BoNT/E positive nonfillet samples has implications for fish handling. Caution should be used when dealing with this fraction of the fish. It is possible that during the standard filleting procedure, toxin could be liberated from the gut or organs, where it may be present. To minimize the chance of exposure, it is recommended that rubber gloves be worn and the filleting area be thoroughly cleaned following the procedure.

Although BoNT/E was detected in two round goby fillets, there is minimal associated public health risk. In the Great Lakes, round gobies are not considered a sport fish and are not commercially harvested. Rainbow trout, walleye, and perch are considered key sport fish species, and perch and walleye are conventionally harvested by netting. When these species are exhibiting clinical signs of botulism, their abnormal behavior makes them less likely to actively consume or pursue bait used by anglers. These three key commercial fish species are good representatives of fish consumed by humans. At the doses tested, these species had no detectable toxin in their fillet fraction. This fact suggests that the risk associated with consumption of commercially harvested fish is likely very, very low.

However, if fillets did contain BoNT/E, the toxin is easily denatured at high temperatures and would be rendered inactive if the fish were cooked properly (12). In the worst-case scenario, should a BoNT/E laden fish be improperly cooked or eaten raw, the public health risk is still minimal. The estimated lethal dose of BoNT/E for humans is between 1,500 and 2,500 MLD kg-1 (6). Thus, for a 65$\mathrm{kg}$ person, this would be between 97,500 to 162,000 MLD. It is highly unlikely that any fish would be captured alive with this body burden of free toxin. Furthermore, humans have been reported to recover from a dose as high as 250,000 MLD (4).

Implications to predatory birds eating live fish. Unlike humans, birds that eat living fish do not differentiate between fillet and nonfillet samples when consuming their prey. As a result, free toxin present in either body compartment of the fish will find its way into the stomach of the bird predator. Because many fish in our study were alive, even when harboring free toxin (i.e., in the positive BoNT/E nonfillet samples), our results suggest that similarly affected wild fish could represent a vector for BoNT/E transfer to fish-eating birds in nature. Other work in our lab $(14,15)$ has confirmed that fish can survive for prolonged periods, even after consuming relatively high-and ultimately lethal-doses of toxin. The present study verifies that at least some of this toxin can be present as free toxin in affected fish, either during the living morbidity phase or after death. This supports at least one possible route by which living fish can transfer free toxin to those predatory birds that might consume them.

Prior to this study, the lack of experimental data on the availability of BoNT/E in the flesh of fresh fish made it difficult to speculate on the public health risks associated with the consumption of fish captured during natural botulism epizootics. This study suggests that the public health risks associated with the consumption of rainbow trout, yellow perch, and walleye are minimal. As a cautionary measure, fish acting abnormally should not be kept for consumption, and proper cooking and cleaning procedures 
should be followed. Furthermore, the discovery of a high percentage of nonfillet samples that were positive for BoNT/E supports the hypothesis that bird predators that exclusively eat live fish can still be exposed to significant quantities of free toxin.

\section{ACKNOWLEDGMENTS}

We gratefully acknowledge Daniel Leclair at Health Canada for assisting with the fish dissections and Environment Canada for providing funding for the project.

\section{REFERENCES}

1. Anonymous. 2004. Diagnosing botulism in fish in the lower Great Lakes, p. 30-35. In Botulism in Lake Erie Workshop Proceedings. Co-sponsored by New York Sea Grant, Ohio Sea Grant, and Pennsylvania Sea Grant. Erie, Pa.

2. Austin, J. W., and B. Blanchfield. 1996. Health Protection Branch Ottawa: detection of Clostridium botulinum and its toxins in suspect foods and clinical specimens. Polyscience Publications, Montreal, Quebec, Canada.

3. Dolman, C. E. 1964. Botulism as a world health problem, p. 5-32. In K. H. Lewis and K. Cassel (ed.), Botulism: proceedings of a symposium. U.S. Department of Health, Education, and Welfare, Cincinnati, Ohio.

4. Dolman, C. E., G. E. Darby, and R. F. Lane. 1955. Type E botulism due to salmon eggs. Can. J. Public Health 46:135-141.

5. Dolman, C. E., and H. Iida. 1963. Type E botulism: its epidemiology, prevention, and specific treatment. Can. J. Public Health 54:293308.

6. Dolman, C. E., and L. Murakami. 1960. Clostridium botulinum type
F with recent observations of other types. J. Infect Dis. 109:107128.

7. Fisheries and Oceans Canada. 2003. Statistical services. Available at: http://www.dfo.mpo.gc.ca/communic/statistics/recreational/great_ lakes/gl1995/gltab_e.htm. Accessed 12 January 2005.

8. Foegeding, M. 1986. Detection and quantification of spore forming pathogens and their toxins, p. 393-423. In M. D. Pierson and N. J. Sterns (ed.), Foodborne microorganisms and their toxins: developing methodology. Marcel Dekker Inc., New York.

9. Friend, M., and C. Franson. 1999. Avian botulism, p. 271-282. In E. Ciganovich (ed.), Field manual of wildlife diseases: general field procedures and diseases of birds. U.S. Government Printing Office, Washington, D.C.

10. Great Lakes Science Center, United States Geological Service. 2005. Commercial fish production-pound and value. Available at: http:// www.glsc.usgs.gov/_files/cfreports/noaa02.txt. Accessed $6 \overline{\text { April }}$ 2005.

11. Kautter, D. A. 1960. Clostridium botulinum type E in smoked fish. J. Food Sci. 29:843-849.

12. Huss, H. H. 1981. Clostridium botulinum type E and botulism. Thesis. Technological Laboratory, Ministry of Fisheries, Technical University, Lyngby, Denmark.

13. Summerfelt, R. C., and L. S. Smith. 1990. Anaesthesia, surgery, and related techniques p. 213-272. In C. B. Schreck and P. B. Moyle (ed.), Methods for fish biology. American Fisheries Society, Bethesda, Md.

14. Yule, A. M., I. K. Barker, J. W. Austin, and R. D. Moccia. Comparative toxicity of Clostridium botulinum type E neurotoxin to Great Lakes fish: implications to avian botulism. J. Wildl. Dis., in press.

15. Yule, A. M., V. Lepage, J. W. Austin, I. K. Barker, and R. D. Moccia. Chronic exposure of the round goby (neogobius melanostomas) to Clostridium botulinum neurotoxin type E. Submitted for publication. 